\title{
Oxidative Stress in Post-Acute Ischemic Stroke Patients: Relevance of Early Intensive Neurorehabilitation
}

\author{
Irene Ciancarelli ${ }^{1,2 *}$, Caterina Pistarini ${ }^{3}$, Antonio Carolei ${ }^{1}$ and Maria Giuliana Tozzi Ciancarelli ${ }^{1}$
}

${ }^{1}$ Department of Medicine, University of L'Aquila, Italy

${ }^{2}$ Nova Salus Nursing and Rehabilitation Center, Italy

${ }^{3}$ Department of Neurorehabilitation, Salvatore Maugeri Foundation, Italy

\begin{abstract}
Oxidative stress was investigated in 10 post-acute stroke patients (median age $76.40 \pm 2.09$ years) assigned to in-hospital neurorehabilitation within 7 days of onset of a first-ever ischemic stroke. Assessments were made before and after neurorehabilitation. Outcome measures were the National Institutes of Health Stroke Scale (NIHSS), the Barthel Index $(\mathrm{BI})$, the Katz Index $(\mathrm{KI})$, the modified Rankin Scale (mRS) and the modified PULSES profile. Differences between scores evaluated before and after neurorehabilitation were expressed as $\Delta$ scores. Oxidative stress was assessed by measuring plasma amount of total peroxides by-products, nitrite/nitrate metabolites $\left(\mathrm{NO}_{\mathrm{x}}\right)$, total plasma antioxidant capacity (TEAC), Cu/Zn Superoxide Dismutase (Cu/ZnSOD), and serum urate concentration. Ten healthy volunteers (median age $74.30 \pm 3.03$ years) were recruited as controls for comparison of oxidative markers. Neurorehabilitation was associated with an improvement $(P<0.05)$ of all the outcome measures except the mRS score. $\triangle$ PULSES correlated negatively with $\triangle \mathrm{BI}$ scores $(\mathrm{r}=-0.656 ; P=0.040)$. Higher plasma levels of total peroxidative by-products and $\mathrm{NO}_{x}$ metabolites were found in stroke patients than in controls before neurorehabilitation $(P<0.05)$. Their values decreased in stroke patients after neurorehabilitation and were higher than those measured in controls $(P<0.05)$. TEAC, Cu/Zn SOD and serum urate levels were lower in stroke patients than in controls before neurorehabilitation $(P<0.05)$. Their values increased after neurorehabilitation so that no differences were observed comparing these values with those found in controls. Changes observed before and after neurorehabilitation in plasma NOx amount $(\triangle \mathrm{NOx})$ correlated positively with $\triangle$ NIHSS scores $(\mathrm{r}=0.675 ; P=0.032)$ and negatively with $\triangle \mathrm{PULSES}(\mathrm{r}=-$ $0.845 ; P=0.002$ ). Cu/ZnSOD changes found before and after neurorehabilitation ( $\Delta \mathrm{Cu} / \mathrm{Zn}$ SOD) and $\Delta \mathrm{KI}$ correlated positively $(r=0.713 ; P=0.021)$. Our results showed that intensive neurorehabilitation may modulate oxidative stress and suggest its effectiveness in redox-mediated plasticity processes involved in the recovery of stroke-induced neurological deficits.
\end{abstract}

\section{Introduction}

Cerebral ischemia triggers a highly interconnected cascade of events that interfere with a complex network of cell-cell signalling mechanisms to support new functional adaptive strategies aimed to compensate for stroke-induced neuronal loss and consequent impaired body functions [1,2]. Research advances provide evidence that spontaneous recovery may occur after an ischemic insult and that the brain, regardless of age of stroke survivors, has a certain capacity for self-repair after damage [3,4]. Indeed, synaptic plasticity tends to increase in cerebral tissue adjacent to the necrotic area in order to reorganize residual neuronal circuits and to promote parallel processes of neurovascular remodelling [5]. Several mechanisms may contribute to brain activation patterns after an ischemic insult and are involved in neuronal excitability and plasticity after stroke. Potential regulators of neuronal activity and plasticity after stroke may be represented by the increased generation and release by metabolically active neurons of reactive oxygen species (ROS) and reactive nitrogen species (RNS) [6,7]. Functional neuroimaging studies showed that rehabilitative interventions may contribute to modulate post-stroke brain reorganization by exerting a positive influence over ongoing plasticity processes allowing new functional communications in the remaining neuronal circuits and by enhancing neuronal activity in pre-existing damaged networks $[4,5,8]$. Therefore, stroke rehabilitation could be recognized as a valid approach in translating the mechanisms of neuronal plasticity into strategies promoting functional recovery from neurologic lesions. Besides, it is widely accepted that intensive neurorehabilitation should begin as soon as possible after stroke [9]. Studies assessing whether intensive neurorehabilitation might interfere with ROS/RNS-mediated signalling mechanisms involved in post-stroke-induced synaptic remodelling processes and neuronal excitability, are heterogeneous because of differences in study protocols and biomarkers measured [10-12]. The aim of the present study was to investigate the extent of stroke-mediated oxidative stress in patients within a few days after stroke onset and the possible effectiveness of an early in-hospital intensive neurorehabilitation on the modulation of some oxidative markers as potential expression of neuronal plasticity and self-repair capacity for the recovery of motor performances.

\section{Material and Methods}

\section{Patients selection and evaluation}

Ten post-acute stroke patients ( 6 females and 4 males; median age \pm SD $76.40 \pm 2.09$ years), assigned to in-hospital neurorehabilitation in the Nova Salus Rehabilitation Center (NSRC) were recruited from January to April 2013. Stroke patients were enrolled within 7 days of the onset of a first-ever ischemic stroke confirmed by brain computed tomography or magnetic resonance imaging. Alert stroke patients with

*Corresponding author: Irene Ciancarelli, MD, Assistant Professor of Neurorehabilitation, Department of Medicine, Health, and Environmental Sciences, University of L'Aquila, Italy, Tel: +39.0862 .368542; Fax: +39.0862.368542; E-mail: irene.ciancarelli@univaq.it

Received July 02, 2013; Accepted July 17, 2013; Published July 25, 2013

Citation: Ciancarelli I, Pistarini C, Carolei A, Ciancarelli MGT (2013) Oxidative Stress in Post-Acute Ischemic Stroke Patients: Relevance of Early Intensive Neurorehabilitation. J Neurol Neurophysiol 4: 154. doi:10.4172/2155-9562.1000154

Copyright: (c) 2013 Ciancarelli I, et al. This is an open-access article distributed under the terms of the Creative Commons Attribution License, which permits unrestricted use, distribution, and reproduction in any medium, provided the original author and source are credited. 
right or left hemiparesis and able to control the trunk in the upright and sitting position without the help of their arms for $10-20 \mathrm{sec}$ were considered eligible. Exclusion criteria were: chronic pathologic conditions potentially able to influence systemic redox equilibrium, current treatment with statins and antioxidants before the index stroke; pre-stroke walking disability; any disease preventing the participation in a regular exercise program, as indicated by medical examination to allow neuromotor rehabilitation. Patient stroke severity was evaluated according to the National Institutes of Health Stroke Scale (NIHSS); disability with the Barthel Index (10-item) (BI), the Katz Index of Independence in Activities of Daily Living (6-item) (KI), and the modified Rankin Scale (7-item) (mRS) [13-16].-The modified PULSES profile (24-item), an acronym for 6 functions and factors, has been used to predict rehabilitation potential and to monitor neurorehabilitative patient progress [17]. Ten apparently healthy volunteers ( 3 females, 7 males; median age \pm SD $74.30 \pm 3.03$ years) were enrolled in the study as control group for comparison of oxidative stress markers. Detailed information on lifestyle and medication used by both stroke patients and controls were recorded. Blood samples for laboratory routine analyses and oxidative stress markers were withdrawn from each participant to the study. In stroke patients all the measured parameters were evaluated before and at the end of the intensive neurorehabilitation program.

The study design, approved by the Local Ethic Committee and in accordance with the Declaration of Helsinki, was explained in advance to all participants or to their caregivers who gave their written informed consent.

\section{Neurorehabilitation protocol}

Details of the scheduled intensive neurorehabilitation protocol including conventional neuromotor rehabilitation and occupational therapy are reported in a previous paper [12].

\section{Blood sampling}

Venous blood samples with or without anticoagulant were drawn from all subjects in a fasting state and centrifuged to obtain plasma or serum used for routine laboratory analysis and measurements of oxidative stress markers. In the case of oxidative analyses, plasma and serum samples were frozen at $-80^{\circ} \mathrm{C}$ until analyses were performed.

\section{Laboratory analysis}

Among routine parameters, glucose, total cholesterol, triglycerides, high density lipoprotein-cholesterol (HDL-cholesterol), low density lipoprotein-cholesterol (LDL-cholesterol) were analyzed using AbbottDiagnostic Clinical Chemistry Tests, Architect c8000 (Latina, Italy). Creatinine levels $(\mathrm{ng} / \mathrm{ml})$ were measured in urine samples using a colorimetric assay (Cayman Chemical Company, Ann Arbor, MI, USA) with a sensitivity of $0.01 \mathrm{mg} / \mathrm{ml}$, an intra-assay CV of $2.7 \%$ and an inter-assay $\mathrm{CV}$ of $3.0 \%$.

\section{Oxidative stress markers}

Levels of nitrite/nitrate $\left(\mathrm{NO}_{\mathrm{x}}\right)$ final metabolites in plasma $(\mu \mathrm{mol} / \mathrm{l})$, as an expression of $\mathrm{NO}$ availability, were evaluated as previously reported [18]. Plasma total peroxide concentrations $(\mu \mathrm{mol} / \mathrm{l})$, total plasma antioxidant capacity (TEAC) $(\mathrm{mmol} / \mathrm{l}), \mathrm{Cu} / \mathrm{Zn}$ Superoxide Dismutase $(\mathrm{Cu} / \mathrm{Zn} \mathrm{SOD})$ plasma levels $(\mathrm{ng} / \mathrm{ml})$, and uric acid plasma levels $(\mathrm{mg} / \mathrm{dl})$ were assayed as described in a previous paper [19].

\section{Statistical Analysis}

All data are given as means \pm standard error of the mean (S.E.M.).
Non parametric Mann-Whitney rank sum test was used to compare differences of pre- and post- neurorehabilitation treatment in stroke patients $v s$. controls. Wilcoxon matched-pairs signed rank test was used to compare before and after neurorehabilitation treatment values, and to compare the scores of the different rating scales. Pearson's correlation analysis was performed to determine the relationship between variables of interest. Statistical significance was accepted if $P<0.05$. All statistical analyses were performed with SPSS, version 16 .

\section{Results}

The clinical characteristics of post-acute stroke patients and controls are reported in Table 1 . Stroke patients were assigned to in-hospital neurorehabilitation within 7 days of onset of first-ever ischemic stroke. No differences were observed between stroke patients and controls in age, blood pressure, glucose, and BMI, while differences $(P<0.05)$ were found in plasma values of total cholesterol $(181 \pm 9.7 v s$. $168.7 \pm 4.7 \mathrm{mg} / \mathrm{dl})$, triglycerides $(105 \pm 5.4$ vs. $70 \pm 3.4 \mathrm{mg} / \mathrm{dl})$, LDL-C $(140 \pm 4.3$ vs. $115 \pm 4.2 \mathrm{mg} / \mathrm{dl})$, and HDL-C $(65 \pm 5.2$ vs. $51 \pm 7.6)$. Unchanged values of these parameters were observed at the end of neurorehabilitation. All stroke patients completed the rehabilitative program with $100 \%$ overall compliance and without any adverse event. Intensive neurorehabilitation was associated with a significant improvement $(P<0.05)$ of all clinical and functional outcome measures except the modified Rankin Scale score (Table 2). $\triangle$ PULSES correlated negatively with $\Delta \mathrm{BI}$ scores $(\mathrm{r}=-0.656 ; P=0.040)$. The values of oxidative stress markers in stroke patients before and after neurorehabilitation and in controls were reported in Table 3. Higher plasma levels of total peroxidative by-products and $\mathrm{NO}_{x}$ metabolites were found in stroke patients than in controls before neurorehabilitation $(P<0.05)$. Their values decreased $(P<0.05)$ in stroke patients after neurorehabilitation but were higher than those measured in controls. Changes observed before and after neurorehabilitation in plasma NOx amount $(\triangle \mathrm{NOx})$ correlated positively with $\triangle$ NIHSS scores $(\mathrm{r}=0.675 ; P=0.032)$ and negatively with $\triangle$ PULSES scores $(r=-0.845 ; P=0.002)$. TEAC, $\mathrm{Cu} /$ $\mathrm{Zn}$ SOD and serum urate levels were lower in stroke patients than in controls before neurorehabilitation $(P<0.05)$. Their values increased after neurorehabilitation so that no differences were observed when comparing these values with those found in controls. Changes observed before and after neurorehabilitation in $\mathrm{Cu} / \mathrm{Zn}$ SOD $(\Delta \mathrm{Cu} / \mathrm{Zn}$ SOD $)$ and in $\triangle \mathrm{KI}$ correlated positively $(\mathrm{r}=0.713 ; P=0.021)$.

\section{Discussion}

Over-generation of free radicals and decreased antioxidant

\begin{tabular}{|c|c|c|c|}
\hline & Stroke Patients & Controls & \\
\hline Gender (Men/Women) & $4 / 6$ & $7 / 3$ & \\
\hline Age (Years) & $76.40 \pm 2.09$ & $74.30 \pm 3.03$ & \\
\hline & Before-rehabilitation & After-rehabilitation & \\
\hline BMl $\left(\mathrm{kg} / \mathrm{m}^{2}\right)$ & $25.65 \pm 2.4$ & $25.70 \pm 1.8$ & $25.13 \pm 2.9$ \\
\hline SBP $(\mathrm{mmHg})$ & $140.57 \pm 6.18$ & $132.62 \pm 6.57$ & $134.20 \pm 5.54$ \\
\hline DBP $(\mathrm{mmHg})$ & $85.40 \pm 2.80$ & $80.30 \pm 3.10$ & $76.45 \pm 2.38$ \\
\hline Glucose $(\mathrm{mg} / \mathrm{dl})$ & $104 \pm 4.2$ & $96 \pm 2.5$ & $102 \pm 1.8$ \\
\hline Total cholesterol $(\mathrm{mg} / \mathrm{dl})$ & $180.6 \pm 9.68^{*}$ & $185.2 \pm 2.1^{*}$ & $168.7 \pm 8.64$ \\
\hline Triglycerides $(\mathrm{mg} / \mathrm{dl})$ & $105.73 \pm 0.54^{*}$ & $102.83 \pm 0.60^{*}$ & $72.20 \pm 3.04$ \\
\hline HDL-C $(\mathrm{mg} / \mathrm{dl})$ & $65.8 \pm 5.2^{*}$ & $62.6 \pm 2.1^{*}$ & $51.6 \pm 7.6$ \\
\hline LDL-C $(\mathrm{mg} / \mathrm{dl})$ & $140.3 \pm 4.3^{*}$ & $137.2 \pm 2.6^{*}$ & $115 \pm 4.2$ \\
\hline
\end{tabular}

BMI: body mass index; SBP: systolic blood; DBP: diastolic blood pressure; HDL-C: High-density lipoprotein-cholesterol; LDL-C: low density lipoproteincholesterol. ${ }^{*} P<0.05$ vs. control values.

Table 1: Clinical parameters in post-acute stroke patients before and after neurorehabilitation and controls. 


\begin{tabular}{|c|c|c|c|}
\hline & Before- rehabilitation & After- rehabilitation & $\boldsymbol{\Delta}$ scores \\
\hline NIHSS scores & $9.50 \pm 1.38$ & $6.40 \pm 1.20^{*}$ & $3.10 \pm 0.57$ \\
\hline mRS & $4.29 \pm 0.16$ & $4.07 \pm 0.27$ & $0.22 \pm 0.15$ \\
\hline $\mathrm{BI}$ & $43.50 \pm 10.28$ & $65.00 \pm 10.49^{*}$ & $-21.5 \pm 0.44$ \\
\hline KI & $5.20 \pm 1.45$ & $7.50 \pm 1.44^{*}$ & $-2.30 \pm 0.30$ \\
\hline PULSES & $16.29 \pm 1.62$ & $11.40 \pm 1.98^{*}$ & $4.89 \pm 0.12$ \\
\hline
\end{tabular}

${ }^{*} P<0.05$ before $v s$. after values NIHSS, National Institutes of Health Stroke Scale (11-item); mRS, modified Rankin Scale (7-item); BI, Barthel Index (10-item); KI, Katz Index (6-item); PULSES profile (24-item); $\Delta$ scores, difference of values evaluated before and at the end of rehabilitation.

Table 2: Outcome measures evaluated before and after neurorehabilition

\begin{tabular}{|c|c|c|c|}
\hline & \multicolumn{2}{|c|}{ Stroke Patients } & Controls \\
\hline & $\begin{array}{c}\text { Before- } \\
\text { rehabilitation }\end{array}$ & After-rehabilitation & \\
\hline Total peroxides $(\mu \mathrm{mol} / \mathrm{l})$ & $517.25 \pm 47.43^{*}$ & $331.96 \pm 22.17^{\star \circ \circ}$ & $144.7 \pm 12.1$ \\
\hline $\mathrm{NO}_{\mathrm{x}}$ metabolites $(\mu \mathrm{mol} / \mathrm{l})$ & $26.62 \pm 2.04^{*}$ & $16.44 \pm 1.3^{* \circ}$ & $13.1 \pm 0.6$ \\
\hline $\mathrm{Cu} / \mathrm{ZnSOD}(\mathrm{ng} / \mathrm{ml})$ & $32.4 \pm 1.6^{*}$ & $44.2 \pm 0.7^{\circ \circ}$ & $46.6 \pm 1.2$ \\
\hline Uric acid $(\mathrm{mg} / \mathrm{dl})$ & $3.7 \pm 0.08^{*}$ & $5.08 \pm 0.12^{\circ \circ}$ & $5.03 \pm 0.17$ \\
\hline TEAC $(\mathrm{mmol} / \mathrm{l})$ & $0.65 \pm 0.08^{*}$ & $1.08 \pm 0.16^{\circ \circ}$ & $1.31 \pm 0.21$ \\
\hline
\end{tabular}

${ }^{*} P<0.05$ vs. control values ${ }^{\circ} P<0.05$ vs. before-rehabilitation

Table 3: Oxidative stress markers stroke patients before and after neurorehabilitation and in controls.

defences have long been considered as mediators of stroke-related events and oxidative damage of the brain which is particularly sensitive to redox unbalance [20]. Emerging evidence suggests that controlled concentrations of ROS and NO, because of their physical/chemical characteristics, may assume the significance of signalling molecules that promote new communications in the remaining neuronal circuits and, as a consequence, may be pivotal in controlling plastic processes and subtending functional recovery $[6,19,21]$. Recovery of body functions and activities is a complex process that occurs through a combination of spontaneous recovery of damaged neural tissue, reorganization of neural pathways and learning-dependent processes $[8,9]$. Available studies suggest that the creation of specific and repetitive learning situations facilitates and promotes mechanisms of neural plasticity and that task-oriented training may assist the natural pattern of functional recovery [22,23]. Functional neuroimaging studies show that neurorehabilitation represents a cornerstone approach that facilitates post-stroke recovery by translating neuronal plasticity into sophisticated strategies that modify cerebral activation patterns $[5,24,25]$. It is widely accepted that a better outcome of rehabilitation is achieved with an early initiation of treatment, aimed to provide a multimodal, intense, and carefully individualized program that calibrates the complexity of rehabilitative protocol to disability as assessed in each patient [26]. Besides, assessment of rehabilitation effectiveness through outcome measures of different types is basilar to better define neurologic deficits, to monitor the effects of treatment and natural recovery, and to understand the relationship between reductions in disability and improvements induced by therapeutic strategies. Accordingly, findings of the present study confirmed the relevance and the effectiveness of early neurorehabilitation in clinical outcome of post-acute stroke patients as showed by a global improvement of all the outcome measures except the mRS scores. Indeed, decreased values of NIHSS scores suggested an improvement of the neurological deficits and of the related disability, and increased values of BI and of $\mathrm{KI}$ scores suggested a significant improvement in the overall functional autonomy of stroke patients. Notably, the correlation that we found between values of the BI scores and PULSES profile, evaluated before and after neurorehabilitation, may represent a valid and predictive measure for assessing desirable realistic goals and disability improvement in the stroke rehabilitative setting. The hypothesis of an involvement of oxidative stress in the rehabilitative-induced ongoing process subtending brain recovery is supported by the evidence that 6-week intensive neurorehabilitation in stroke patients within 7 days of onset of first-ever ischemic stroke is effective in modulating oxidative markers in relation to the recovery of neurological deficits, as a possible cause or effect of neuronal plasticity. As indicated by our results, higher plasma levels of total peroxidative by-products and $\mathrm{NO}_{x}$ metabolites, as a global expression of stroke-induced systemic pro-oxidative status, were found before neurorehabilitation in stroke patients than in controls. Their values decreased at the end of inpatient neurorehabilitation even if they remained higher than those measured in controls. Besides, correlations between changes of NIHSS and PULSES scores and of plasma NO metabolites evaluated before and after neurorehabilitation, suggested that NO is involved as a likely fast messenger of brain activation patterns in neuronal processes promoted by neurorehabilitation and that it may influence recovery after stroke. A further evidence of intensive neurorehabilitation effectiveness in promoting neuronal activity and plasticity through modulation of redox balance is also supported, in our opinion, by the modifications of antioxidant defences before and after rehabilitative treatment. Indeed, TEAC and $\mathrm{Cu} / \mathrm{Zn}$ SOD and serum urate levels were lower before neurorehabilitation in stroke patients than in controls, but their values increased after neurorehabilitation to reach levels similar to those found in controls. Interestingly, the correlation we found between the differences of values of $\mathrm{Cu} / \mathrm{ZnSOD}$ levels and the KI scores measured before and after neurorehabilitation underlines that the efficient defence mechanism against superoxide radicals provided by $\mathrm{Cu} /$ $\mathrm{Zn}$-SOD isozyme is strategically important for plasticity phenomena leading to the improvement in the overall functional autonomy of stroke patients. Therefore, it is presumable that redox balance could assume a strategic role that concurs to the post-stroke spontaneous patterns of plasticity process providing an adequate environment for rehabilitation-associated mechanisms subtending functional recovery. Further investigations should be carried out to validate the findings of the present study by an adequately powered multicenter case-control study aimed to investigate a larger sample of stroke survivors including patients with a history of cardio- and cerebrovascular events before the index stroke.

\section{References}

1. Arai K, Lok J, Guo S, Hayakawa K, Xing C, et al. (2011) Cellular mechanisms of neurovascular damage and repair after stroke. J Child Neurol 26: 1193-1198.

2. Candelario-Jalil E (2009) Injury and repair mechanisms in ischemic stroke: considerations for the development of novel neurotherapeutics. Curr Opin Investig Drugs 10: 644-654.

3. Zorowitz R, Brainin M (2011) Advances in brain recovery and rehabilitation 2010. Stroke 42: 294-297.

4. Eliassen JC, Boespflug EL, Lamy M, Allendorfer J, Chu WJ, et al. (2008) Brainmapping techniques for evaluating poststroke recovery and rehabilitation: a review. Top Stroke Rehabil 15: 427-450.

5. Dijkhuizen RM, van der Marel K, Otte WM, Hoff El, van der Zijden JP, et al. (2012) Functional MRI and Diffusion Tensor Imaging of Brain Reorganization After Experimental Stroke. Transl Stroke Res 3: 36-43.

6. Dickinson BC, Peltier J, Stone D, Schaffer DV, Chang CJ (2011) Nox2 redox signaling maintains essential cell populations in the brain. Nat Chem Biol 7: 106-112.

7. Cheng A, Hou Y, Mattson MP (2010) Mitochondria and neuroplasticity. ASN Neuro 2: e00045.

8. Di Filippo M, Tozzi A, Costa C, Belcastro V, Tantucci M, et al. (2008) Plasticity and repair in the post-ischemic brain. Neuropharmacology 55: 353-362. 
Citation: Ciancarelli I, Pistarini C, Carolei A, Ciancarelli MGT (2013) Oxidative Stress in Post-Acute Ischemic Stroke Patients: Relevance of Early Intensive Neurorehabilitation. J Neurol Neurophysiol 4: 154. doi:10.4172/2155-9562.1000154

9. Langhorne P, Bernhardt J, Kwakkel G (2011) Stroke rehabilitation. Lancet 377 : 1693-1702.

10. Manolescu BN, Berteanu M, Oprea E, Chiriac N, Dumitru L, et al. (2011) Dynamic of oxidative and nitrosative stress markers during the convalescent period of stroke patients undergoing rehabilitation. Ann Clin Biochem 48: 338343

11. Unno Y, Katayama M, Shimizu H (2010) Does functional outcome in acute ischaemic stroke patients correlate with the amount of free-radical scavenger treatment? A retrospective study of edaravone therapy. Clin Drug Investig 30: 143-155.

12. Ciancarelli I, Di Massimo C, De Amicis D, Carolei A, Tozzi Ciancarelli MG (2012) Evidence of redox unbalance in post-acute ischemic stroke patients. Curr Neurovasc Res 9: 85-90.

13. Wityk RJ, Pessin MS, Kaplan RF, Caplan LR (1994) Serial assessment of acute stroke using the NIH Stroke Scale. Stroke 25: 362-365.

14. MAHONEY FI, BARTHEL DW (1965) FUNCTIONAL EVALUATION: THE BARTHEL INDEX. Md State Med J 14: 61-65.

15. Bonita R, Beaglehole R (1988) Recovery of motor function after stroke. Stroke 19: $1497-1500$.

16. Katz S (1983) Assessing self-maintenance: activities of daily living, mobility, and instrumental activities of daily living. J Am Geriatr Soc 31: 721-727.

17. Marshall SC, Heisel B, Grinnell D (1999) Validity of the PULSES profile compared with the Functional Independence Measure for measuring disability in a stroke rehabilitation setting. Arch Phys Med Rehabil 80: 760-765.

18. Tozzi-Ciancarelli MG, Penco M, Di Massimo C (2002) Influence of acute exercise on human platelet responsiveness: possible involvement of exerciseinduced oxidative stress. Eur J Appl Physiol 86: 266-272.

19. Allen CL, Bayraktutan $U$ (2009) Oxidative stress and its role in the pathogenesis of ischaemic stroke. Int J Stroke 4: 461-470.

20. Unno Y, Katayama M, Shimizu H (2010) Does functional outcome in acute ischaemic stroke patients correlate with the amount of free-radical scavenger treatment? A retrospective study of edaravone therapy. Clin Drug Investig 30 143-155.

21. Langhorne P, Coupar F, Pollock A (2009) Motor recovery after stroke: a systematic review. Lancet Neurol 8: 741-754.

22. Kwakkel G, Kollen B, Lindeman E (2004) Understanding the pattern of functional recovery after stroke: facts and theories. Restor Neurol Neurosci 22 281-299.

23. Eliassen JC, Boespflug EL, Lamy M, Allendorfer J, Chu WJ, et al. (2008) Brainmapping techniques for evaluating poststroke recovery and rehabilitation: a review. Top Stroke Rehabil 15: 427-450.

24. Grefkes C, Fink GR (2011) Reorganization of cerebral networks after stroke: new insights from neuroimaging with connectivity approaches. Brain 134 1264-1276.

25. Musicco M, Emberti L, Nappi G, Caltagirone C; Italian Multicenter Study on Outcomes of Rehabilitation of Neurological Patients (2003) Early and long-term outcome of rehabilitation in stroke patients: the role of patient characteristics, time of initiation, and duration of interventions. Arch Phys Med Rehabil 84: 551-558.

26. Xing H, Hao W (2007) Effects of early rehabilitation training on activities of daily living in patient with hemiplegia after stroke. Hubei Sports Science 26:367-369. 